\title{
MRI and Dose Selection in a Phase II Trial of Baricitinib with Conventional Synthetic Disease-modifying Antirheumatic Drugs in Rheumatoid Arthritis
}

\author{
Charles Peterfy, Julie DiCarlo (D, Paul Emery, Mark C. Genovese (D), Edward C. Keystone, \\ Peter C. Taylor, Doug E. Schlichting D, Scott D. Beattie, Monica Luchi, and William Macias
}

ABSTRACT. Objective. Magnetic resonance imaging (MRI) was used in a phase IIb study of baricitinib in patients with RA to support dose selection for the phase III program.

Methods. Three hundred one patients with active RA who were taking stable methotrexate were randomized 2:1:1:1:1 to placebo or once-daily baricitinib $(1,2,4$, or $8 \mathrm{mg})$ for up to 24 weeks. One hundred fifty-four patients with definitive radiographic erosion had MRI of the hand/wrist at baseline and at weeks 12 and 24. Two expert radiologists, blinded to treatment and visit order, scored images for synovitis, osteitis, bone erosion, and cartilage loss. Combined inflammation (osteitis $+3 \times$ synovitis score) and total joint damage (erosion $+2.5 \times$ cartilage loss score) scores were calculated. Treatment groups were compared using ANCOVA adjusting for baseline scores.

Results. Mean changes from baseline to Week 12 for synovitis were $-0.10,-1.50$, and -1.60 for patients treated with placebo, baricitinib $4 \mathrm{mg}$, and baricitinib $8 \mathrm{mg}$, respectively ( $\mathrm{p}=0.003 \mathrm{vs}$ placebo for baricitinib 4 and $8 \mathrm{mg}$ ). Mean changes for osteitis were $0.00,-3.20$, and -2.10 ( $p=0.001$ vs placebo for baricitinib $4 \mathrm{mg}$ and $\mathrm{p}=0.037$ for $8 \mathrm{mg}$ ), respectively. Mean changes for bone erosion were $0.90,0.10$, and $0.40(\mathrm{p}=0.089$ for $4 \mathrm{mg}$ and $\mathrm{p}=0.275$ for $8 \mathrm{mg}$ ), respectively, in these treatment groups.

Conclusion. MRI findings in this subgroup of patients suggest suppression of synovitis, osteitis, and combined inflammation by baricitinib 4 and $8 \mathrm{mg}$. This corroborates previously demonstrated clinical efficacy of baricitinib and increases confidence that baricitinib $4 \mathrm{mg}$ could reduce the radiographic progression in phase III studies. [Clinical trial registration number (www.ClinicalTrials.gov): NCT01185353] (First Release May 15 2019; J Rheumatol 2019;46:887-95; doi:10.3899/ jrheum.171469)

Key Indexing Terms:

RHEUMATOID ARTHRITIS BARICITINIB

From Spire Sciences Inc., Boca Raton, Florida; Division of Immunology and Rheumatology, Stanford University, Stanford, California; Eli Lilly and Co., Indianapolis, Indiana; Incyte Corporation, Wilmington, Delaware, USA; The Leeds Institute of Rheumatic and Musculoskeletal Medicine, University of Leeds, Leeds; Botnar Research Centre, University of Oxford, Oxford, UK; Department of Rheumatology, University of Toronto, Toronto, Ontario, Canada.

This study was funded by Eli Lilly and Co. and Incyte Corp., which jointly developed baricitinib. Dr. DiCarlo was an employee of Spires Science Inc. at the time of this study. Dr. Luchi was an employee of Incyte Corp. at the time of this study. Dr. Macias was an employee of Eli Lilly and Co. at the time of this study.

C. Peterfy, MD, PhD, Spire Sciences Inc.; J. DiCarlo, PhD, Spire Sciences Inc.; P. Emery, MD, FRCP, The Leeds Institute of Rheumatic and Musculoskeletal Medicine, University of Leeds; M.C. Genovese, MD, Division of Immunology and Rheumatology, Stanford University;

E.C. Keystone, MD, FRCP, Department of Rheumatology, University of Toronto; P.C. Taylor, MA, PhD, FRCP, Botnar Research Centre, University of Oxford; D.E. Schlichting, RN, PhD, Eli Lilly and Co.; S.D. Beattie, PhD, Eli Lilly and Co.; M. Luchi, MD, FACR, MBA, Incyte Corp.; W. Macias, MD, PhD, Eli Lilly and Co.

Address correspondence to Dr. C. Peterfy, Spire Sciences Inc.,

5314 Boca Marina Circle N, Boca Raton, Florida 33487, USA.

E-mail: charles.peterfy@spiresciences.com

Full Release Article. For details see Reprints and Permissions at jrheum.org Accepted for publication January 4, 2019.

\section{MAGNETIC RESONANCE IMAGING JAK INHIBITORS}

Inhibition of structural damage progression is important in delaying or preventing rheumatoid arthritis (RA)-related disability. Contemporary phase III RA clinical programs assess disease-modifying properties of new therapies to ensure that improvements in clinical symptoms of RA are associated with reduced rates of joint damage, which may nonetheless progress because of persistent "silent" subclinical inflammation ${ }^{1}$. However, dose-ranging phase II studies frequently do not include assessment of structural damage as a criterion for dose selection under the assumption that clinical responses, particularly reductions in swollen joint counts and acute-phase reactants, will sufficiently predict disease modification ${ }^{2,3}$.

A major reason for not assessing suppression of structural damage in phase II dose-ranging studies relates to the challenges associated with using conventional radiography for this purpose, which typically requires longer placebo-controlled treatment durations $(\geq 24 \text { weeks })^{4}$ and larger numbers of patients than would be feasible or ethical in dose-ranging studies that, by design, may include ineffective doses in addition to the placebo control.

Personal non-commercial use only. The Journal of Rheumatology Copyright @ $\odot$ 2019. All rights reserved 
Using magnetic resonance imaging (MRI) to assess structural damage may overcome some of these constraints. MRI is more sensitive than radiography in detecting joint damage and is able to visualize synovitis and osteitis that radiography cannot $^{5,6}$. In controlled clinical trials, MRI has been shown to discriminate inhibition of progression of bone erosion and cartilage loss in as little as 12 weeks and suppression of synovitis and osteitis within only 2 weeks of initiating active therapy ${ }^{7,8}$, with patient numbers ( 25-30 patients per arm) that are feasible for phase II studies.

Baricitinib is approved for treatment of moderately to severely active RA in adults in over 50 countries including European countries, Canada, the United States, and Japan. In a placebo-controlled, dose-ranging, phase IIb study, the effects of baricitinib on clinical symptoms and function were assessed in patients with moderately to severely active RA despite treatment with methotrexate (MTX) ${ }^{9}$. That study included an MRI substudy assessing changes in synovitis, osteitis, bone erosion, and cartilage loss in hands and wrists in a subgroup of patients with erosive RA to provide additional data to inform dose selection for phase III studies. The results of both the primary study and MRI substudy (reported here) were used to select baricitinib doses for phase III testing. In phase III studies, improved clinical symptoms and function, and inhibition of the progression of radiographically assessed structural damage, were observed with the selected primary dose of baricitinib (4 $\mathrm{mg}$ once daily) in patients who had active disease despite treatment with conventional synthetic disease-modifying antirheumatic drugs (csDMARD; RA-BUILD) ${ }^{10}$ or MTX (RA-BEAM) ${ }^{11}$, and in patients who were naive to treatment with csDMARD $\left(\right.$ RA-BEGIN) ${ }^{12}$. Herein, we report the results of the MRI substudy of the phase II trial and how the MRI data helped support dose selection for the phase III RA program.

\section{MATERIALS AND METHODS}

Study design and patients. Detailed methods of the primary study [Clinical trial registration number (www.ClinicalTrials.gov): NCT01185353] are described elsewhere ${ }^{9}$ and are briefly summarized here. Men and women (18-75 yrs old, $\mathrm{n}=301)$ with active disease despite MTX treatment were enrolled between October 2010 and August 2011. Active RA was defined as having $\geq 8 / 66$ swollen and $\geq 8 / 68$ tender joints, and a C-reactive protein (CRP) measurement $>1.2$ times the upper limit of normal (ULN) or erythrocyte sedimentation rate > ULN $(28 \mathrm{~mm} / \mathrm{h})$. Patients must have received MTX for $\geq 12$ weeks, with a stable dose of $10-25 \mathrm{mg} /$ week for $\geq 8$ weeks prior to baseline. Key exclusion criteria included history of prior biologic DMARD therapy, serious infection within 6 months of baseline, and malignancy within the prior 5 years.

Patients were randomized 2:1:1:1:1 to receive placebo or baricitinib 1 , 2,4 , or $8 \mathrm{mg}$ orally once daily for 12 weeks. Randomization was stratified by participation in the MRI substudy and geographic region. At Week 12, patients initially assigned to placebo or baricitinib $1 \mathrm{mg}$ were rerandomized (1:1) to baricitinib $2 \mathrm{mg}$ twice daily or baricitinib $4 \mathrm{mg}$ once daily for treatment through Week 24. Patients initially assigned to baricitinib 2, 4, or $8 \mathrm{mg}$ continued the same treatment through Week 24.

MRI substudy. After providing informed consent for the substudy, patients at participating centers had conventional radiographs of the hands/wrists and feet reviewed centrally by an expert radiologist for the presence of erosive disease. Patients with any of the following were excluded from the substudy: estimated glomerular filtration rate $<60 \mathrm{ml} / \mathrm{min}$ based on the Modification of Diet in Renal Disease method, prior allergic reaction to gadolinium-based contrast agents, contraindications to MRI (e.g., claustrophobia, pacemakers, aneurysm clips), or previous/current treatment with denosumab. The study was designed by the sponsor, Eli Lilly and Co., an academic advisory board that included non-Lilly authors of this manuscript, and Incyte Corp. The study was conducted in accordance with ethical principles of the Declaration of Helsinki and Good Clinical Practice guidelines, and was approved by the Schulman IRB (\#10-5255-0). Ethics approval was also obtained for all 69 sites. All patients provided written informed consent.

MRI assessments. Patients with $\geq 1$ definitive radiographic erosion had MRI of the most severely involved hand/wrist (determined by highest swollen/tender joint count at baseline) at baseline, Week 12, and Week 24. Patients who discontinued the study early had their next scheduled assessment performed off-schedule, prior to withdrawal. Metacarpophalangeal and proximal interphalangeal joints were scanned separately from the wrist joints (carpometacarpal, intercarpal, and distal radioulnar) using surface coils and 1.5-Tesla whole-body MRI systems. Pulse sequences included coronal, fat-suppressed, T1-weighted 3-D gradient-echo scans before and after intravenous gadolinium contrast and coronal short-tau inversion recovery scans. An acrylic hand frame (M-frame, Spire Sciences Inc.) and biplanar alignment of coronal slices were used to ensure reproducible cross-sectional anatomy on serially acquired MR images. As described in a charter approved prior to image reading, all images were read independently by 2 expert radiologists blinded to treatment assignment and visit order. Images were read in 2 separate sessions: (1) baseline and 12-week images were read simultaneously, but in random order; (2) three months later, baseline and 12-week images were read again, together with the 24-week images, in random order, without knowledge of the prior reading results.

Synovitis, osteitis, and bone erosion were scored using Outcome Measures in Rheumatology Clinical Trials RA MRI scoring (RAMRIS) ${ }^{13}$, and cartilage loss was scored with the validated 9-point Cartilage Loss Scale $\left(\right.$ CARLOS) ${ }^{14}$. Scale-adjusted scores for combined inflammation (osteitis score $+3 \times$ synovitis score) and total joint damage (erosion score $+2.5 \times$ cartilage loss score) were calculated. We multiplied synovitis by 3 to adjust for the about 3 -fold difference between the scales for synovitis (0-24) and for osteitis (0-75). We also adjusted for scale difference when computing total damage score (bone erosion scale ranges $0-250$, cartilage loss ranges $0-100)$.

Statistical analysis. Analyses of data from this study were prespecified in the study's statistical analysis plan. All patients with a baseline MRI score were included in the analyses of inflammation endpoints (synovitis, osteitis, combined inflammation); for joint damage endpoints (erosion, cartilage loss, total joint damage), patients were included if they had baseline and $\geq 1$ postbaseline score. Analysis results presented herein are based on scores obtained in the second MRI reading session unless patients had data from only the first session (i.e., no data after Week 12). Interreader agreement was expressed as intraclass correlation coefficient. The top 5\% of patients with discrepancies in change scores between the 2 readers for synovitis, osteitis, bone erosion, or cartilage loss were adjudicated by consensus review to correct potential input errors. Adjudicated scores replaced the originally discrepant scores. Scores between the 2 readers were otherwise averaged.

Missing Week 12 or 24 MRI scores were imputed by last observation carried forward (LOCF) for synovitis, osteitis, and combined inflammation, thereby assuming that patients who discontinued early would experience no further improvement of these conditions had they continued assigned treatment. Missing scores for bone erosion, cartilage loss, and total joint damage were imputed by linear extrapolation (LE) from baseline and last available scores, thereby assuming a constant rate of progression over time until the imputed timepoint. This method has been endorsed as providing an accurate estimation of mean radiographic change estimates in $\mathrm{RA}^{15}$.

Personal non-commercial use only. The Journal of Rheumatology Copyright @ 2019. All rights reserved. 
To estimate what might have been expected had the placebo and baricitinib 1-mg groups continued past Week 12, Week 24 scores for these treatment arms were extrapolated from the first 12 weeks using these imputation methods as projections of expected results. Other clinical trials using MRI in patients with RA have used similar methods of imputation and extrapolation ${ }^{16}$.

Posthoc sensitivity analyses for mean changes from baseline were conducted using alternative methods to LOCF and LE imputation. Because patients had at most 1 postbaseline observation per analysis period (i.e., placebo-controlled first 12 weeks and second 12 weeks), any scores obtained from early discontinuation scans were discarded. Multiple imputation regression based on prior scheduled timepoints (including baseline) by treatment group was conducted to obtain 20 datasets with no missing values prior to the analysis step. By ignoring collected data on patients who discontinued early and using baseline scores to impute postbaseline scores based on similarity to other randomized patients with complete data, this method was expected to confirm the general direction of findings from the primary analysis, but with less discriminatory power.

The Week 12 and Week 24 changes from baseline for synovitis, osteitis, bone erosion, cartilage loss, combined inflammation, and total joint damage were compared between each baricitinib group and placebo using separate ANCOVA models for each outcome and timepoint, adjusting for baseline scores. Posthoc analyses following multiple imputation were combined using SAS procedure MIANALYZE to obtain appropriate least squares mean estimates and standard errors for assessing statistical significance. To account for variability in the reading and scoring process to identify meaningful changes at the individual patient level, the smallest detectable changes (SDC) for synovitis, osteitis, bone erosion, and cartilage loss were determined by the method of Bruynesteyn, et al ${ }^{17}$.

Two-sided $\mathrm{p}$ values with no adjustment for multiple comparisons were computed for evaluation of statistical significance. Because this was an exploratory and hypothesis-generating substudy, a liberal approach was taken regarding the type I error rate so as not to be limited by statistical power in selecting baricitinib doses for phase III development, even if perhaps some of the conclusions on inhibitory effects were incorrect or overstated. We considered this an acceptable risk that would be brought to light in phase III confirmatory studies. Therefore, by protocol, any results with $\mathrm{p}$ values $<0.20$ and favoring baricitinib (equivalent to 1 -sided $\mathrm{p}$ values $<0.10$ ) were considered significant; however, in this report, significant $\mathrm{p}$ values are defined using the traditional 2 -sided 0.05 threshold, with larger 1 -sided $\mathrm{p}$ values $<0.10$ indicated for reference.

Statistical methods for the primary study results are reported in Keystone, et al ${ }^{9}$.

\section{RESULTS}

Of the 301 patients randomized in the primary study, 205 signed informed consent for the MRI substudy and 154 met entry criteria for documented erosive disease by radiograph and had obtained baseline MRI. Baseline demographic and disease characteristics of patients in the MRI substudy were generally well balanced across the randomized treatment groups (Table 1) and were consistent with those of the overall patient population (data not shown) ${ }^{9}$. After randomization, $139 / 154$ patients remained in the study for the primary Week 12 assessments and 69/79 patients randomized to the 2-, 4-, or 8-mg dose groups with 24-week treatment periods remained through the completion of the MRI substudy. The number of patients with observed, imputed, and analyzed MRI scores are summarized in Table 2. Interreader agreement (expressed as intraclass correlation coefficients) at Week 24 were 0.92 for synovitis, 0.95 for osteitis, 0.95 for bone erosion, and 0.98 for cartilage loss.

In the primary study, statistically significant, dose-dependent improvements in clinical symptoms and function were observed for baricitinib. Treatment effects appeared to

Table 1. Baseline demographic and disease characteristics.

\begin{tabular}{|c|c|c|c|c|c|}
\hline \multirow[t]{2}{*}{ Characteristics } & \multirow[t]{2}{*}{ Placebo, $n=48$} & \multicolumn{4}{|c|}{ Baricitinib } \\
\hline & & $1 \mathrm{mg}, \mathrm{n}=27$ & $2 \mathrm{mg}, \mathrm{n}=29$ & $4 \mathrm{mg}, \mathrm{n}=26$ & $8 \mathrm{mg}, \mathrm{n}=24$ \\
\hline Age, yrs & $48(14)$ & $52(12)$ & $53(13)$ & $53(9)$ & $53(10)$ \\
\hline Sex, $\%$ female & 83 & 89 & 86 & 62 & 88 \\
\hline Duration of RA, yrs & $5(4)$ & $5(4)$ & $6(5)$ & $5(5)$ & $6(5)$ \\
\hline CCP-positive, $\%$ & 63 & 63 & 59 & 73 & 46 \\
\hline RF-positive, $\%$ & 67 & 56 & 66 & 85 & 75 \\
\hline MTX dose, mg/week & $15(4)$ & $17(4)$ & $15(4)$ & $16(5)$ & $16(4)$ \\
\hline Patients receiving prednisone, $\%$ & 54 & 56 & 62 & 50 & 58 \\
\hline Tender joint count, 68 & $21(10)$ & $19(9)$ & $27(14)$ & $18(12)$ & $23(10)$ \\
\hline Swollen joint count, 66 & $14(6)$ & $15(6)$ & $18(9)$ & $14(6)$ & $16(8)$ \\
\hline DAS28-hsCRP & $5.5(0.9)$ & $5.4(0.8)$ & $5.6(0.9)$ & $5.1(1.0)$ & $5.7(0.9)$ \\
\hline HAQ-DI & $1.08(0.64)$ & $1.24(0.70)$ & $1.14(0.68)$ & $0.99(0.61)$ & $1.21(0.61)$ \\
\hline hsCRP, mg/l (ULN = $3 \mathrm{mg} / \mathrm{l})$ & $20(31)$ & $12(12)$ & $15(27)$ & $14(22)$ & $14(15)$ \\
\hline $\mathrm{ESR}, \mathrm{mm} / \mathrm{h}$ & 40 (19) & $33(18)$ & $32(11)$ & $35(18)$ & $40(16)$ \\
\hline Synovitis & $7.3(5.8)$ & $6.7(5.2)$ & $6.9(5.6)$ & $6.7(4.8)$ & $7.0(5.0)$ \\
\hline Osteitis & $6.5(9.3)$ & $6.9(8.0)$ & $6.4(8.3)$ & $8.0(11.3)$ & $4.9(5.2)$ \\
\hline Combined inflammation & $28.4(22.8)$ & $27.1(20.3)$ & $27.1(22.7)$ & $28.2(23.3)$ & $25.9(17.5)$ \\
\hline Bone erosion & $11.2(8.4)$ & $12.8(10.0)$ & $15.1(15.8)$ & $11.7(8.4)$ & $11.9(11.5)$ \\
\hline Cartilage loss & $10.3(12.6)$ & $11.2(16.5)$ & $13.1(18.9)$ & $8.5(11.6)$ & $9.1(12.2)$ \\
\hline Total joint damage & $37.0(38.2)$ & $40.7(48.7)$ & $48.0(61.8)$ & $32.9(35.7)$ & $34.6(41.3)$ \\
\hline
\end{tabular}

Values are mean (SD) unless otherwise indicated. CCP: cyclic citrullinated peptide antibodies; DAS28-hsCRP: Disease Activity Score based on 28-joint count and level of high-sensitivity C-reactive protein; ESR: erythrocyte sedimentation rate; HAQ-DI: Health Assessment Questionnaire-Disability Index; MTX: methotrexate; RA: rheumatoid arthritis; RF: rheumatoid factor; ULN: upper limit of normal. 
Table 2. Summary of observed on schedule, early termination, and imputed MRI scores.

\begin{tabular}{|c|c|c|c|c|c|c|}
\hline & & \multirow[t]{2}{*}{ Placebo } & \multicolumn{4}{|c|}{ Baricitinib } \\
\hline & & & $1 \mathrm{mg}$ & $2 \mathrm{mg}$ & $4 \mathrm{mg}$ & $8 \mathrm{mg}$ \\
\hline \multicolumn{7}{|c|}{ Joint damage outcomes (bone erosion, cartilage loss) } \\
\hline \multirow[t]{3}{*}{ Week 12} & Total analyzed & 39 & 25 & 29 & 25 & 24 \\
\hline & Observed on schedule & 38 & 25 & 28 & 24 & 24 \\
\hline & Observed early termination & 1 & 0 & 1 & 1 & 0 \\
\hline \multirow{3}{*}{ Week 24} & Observed on schedule & 0 & 0 & 27 & 19 & 23 \\
\hline & Observed early termination & 39 & 25 & 2 & 6 & 1 \\
\hline & Imputed (LE) & 39 & 25 & 2 & 6 & 1 \\
\hline \multicolumn{7}{|c|}{ Patients with inflammation outcomes (synovitis, osteitis) } \\
\hline Baseline & Observed & 48 & 27 & 29 & 26 & 24 \\
\hline Week 12 & Total analyzed & 48 & 27 & 29 & 26 & 24 \\
\hline \multirow{3}{*}{ Week 24} & Observed on schedule & 0 & 0 & 27 & 19 & 23 \\
\hline & Observed early termination & 39 & 25 & 2 & 6 & 1 \\
\hline & Imputed (LOCF) & 48 & 27 & 2 & 7 & 1 \\
\hline
\end{tabular}

MRI: magnetic resonance imaging; LE: linear extrapolation; LOCF: last observation carried forward.

plateau at the 4-mg dose, with minimal additional improvement with the $8-\mathrm{mg}$ dose $^{9}$. For patients enrolled in the substudy, American College of Rheumatology 20\% (ACR20) response rates were statistically significantly higher for the baricitinib 4-mg and 8-mg doses compared to placebo at Week 12. Declines in Disease Activity Score based on 28-joint count and level of high-sensitivity CRP, Health Assessment Questionnaire-Disability Index (baricitinib $8 \mathrm{mg}$ only), and swollen joint counts were statistically significantly larger for the baricitinib 4-mg and 8-mg doses compared to placebo at Week 12 (Appendix 1). At Week 24, treatment responses for the 4-mg and 8-mg doses appeared to be similar, and both were associated with higher degrees of efficacy compared to the 2-mg dose.

With regard to MRI measures of inflammation, compared to placebo, statistically significant improvements were observed at Week 12 in mean synovitis, osteitis, and combined inflammation scores in patients assigned to baricitinib 4-mg and 8-mg, and in mean synovitis and combined inflammation in the 1-mg arm (Figure 1). At Week 24, mean scores for synovitis, osteitis, and combined inflammation continued to improve; decreases in synovitis and combined inflammation appeared to be dose-dependent. Posthoc sensitivity analyses confirmed these findings, except that they were more robust for $4 \mathrm{mg}$ (all endpoints significant), less so for $8 \mathrm{mg}$ (synovitis and combined inflammation significant), and not so for $1 \mathrm{mg}$ (none significant).

Regarding MRI measures of damage, compared to placebo, statistically significant suppression of progression was noted at Week 12 in the baricitinib 2-mg treatment arm for bone erosion, in the 4-mg arm for cartilage loss, and in the 2-mg and 4-mg arms for total joint damage (Figure 2). At Week 24, bone erosion, cartilage loss, and total joint damage scores remained stable. Posthoc sensitivity analyses confirmed the findings for bone erosion, but not for cartilage loss and total joint damage (no significant effects identified).

Table 3 displays summaries of the percentage of patients with improvements exceeding the SDC for synovitis (Week 12 SDC $=1.8$; Week 24 SDC $=2.1$ ) and osteitis (Week 12 $\mathrm{SDC}=2.5$; Week $24 \mathrm{SDC}=2.3$ ) and with no worsening in bone erosion (Week $12 \mathrm{SDC}=1.6$; Week $24 \mathrm{SDC}=1.8$ ) or in cartilage loss (Week $12 \mathrm{SDC}=1.5$; Week $24 \mathrm{SDC}=1.8$ ). Additionally, the table displays the mean percentages of uninvolved joints (per patient) that developed new synovitis or cartilage loss and uninvolved bones that developed new osteitis or erosions. At Week 12, higher rates of improvement in synovitis were observed in the baricitinib 1-mg, 4-mg, and 8 -mg treatment groups relative to placebo and in osteitis in all dose groups; a higher rate of no worsening in bone erosion was observed in the all treatment groups. However, statistical significance was not assessed, and results are considered of a descriptive design only and supportive of the mean change analyses.

\section{DISCUSSION}

The MRI findings from this substudy demonstrate that baricitinib decreased synovitis and osteitis, and inhibited progression of bone erosion and cartilage loss within 12 weeks of initiating treatment in patients with active and erosive RA who previously showed inadequate response to

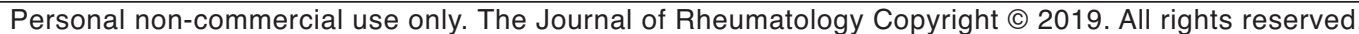


Synovitis - Week 12

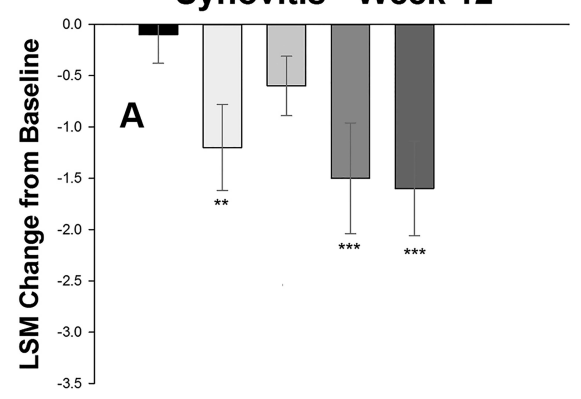

Osteitis - Week 12
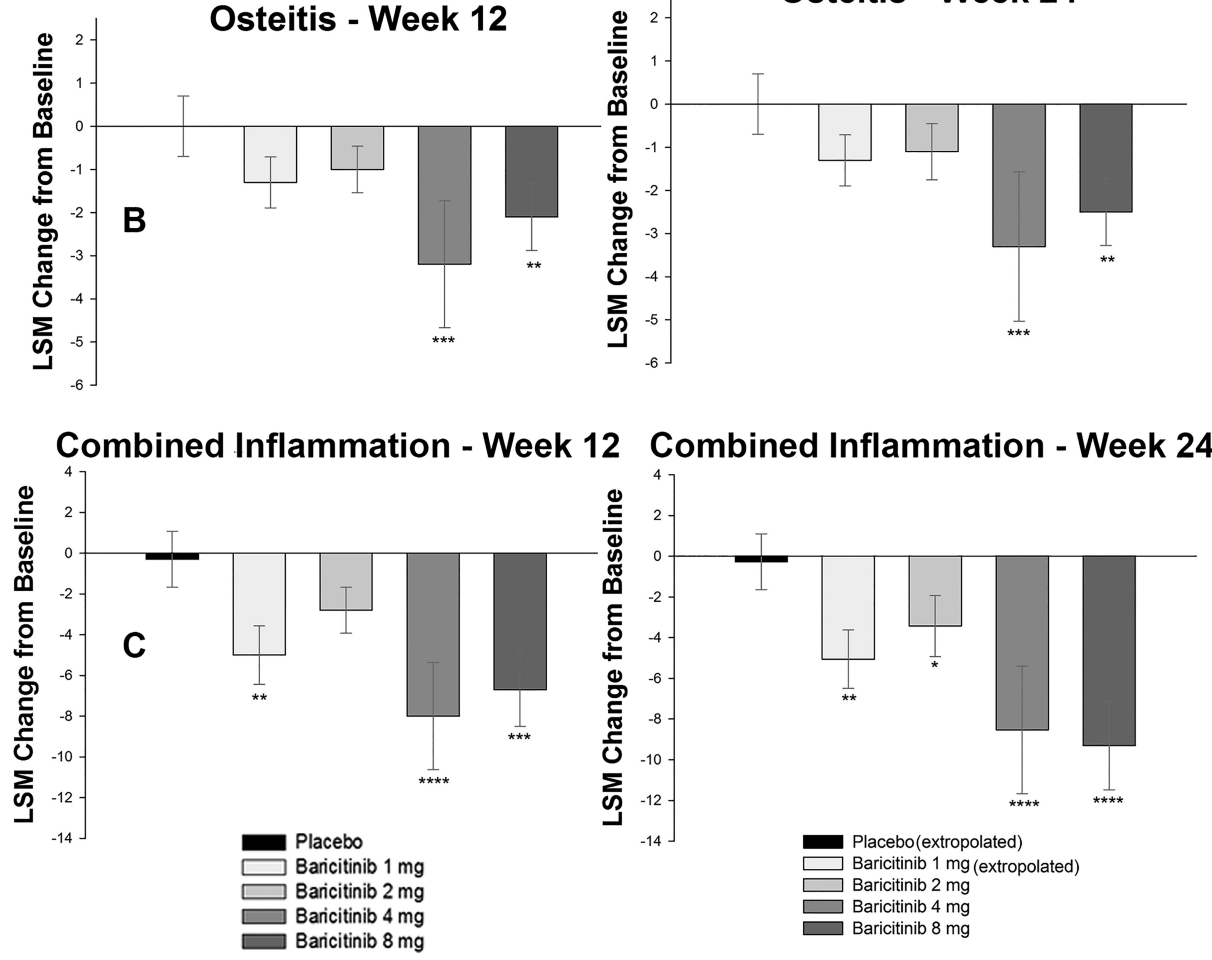

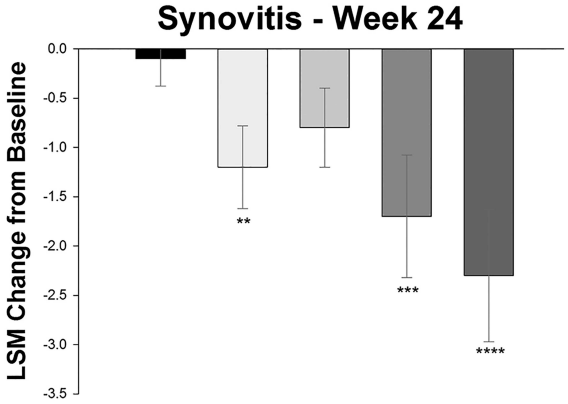

Osteitis - Week 24

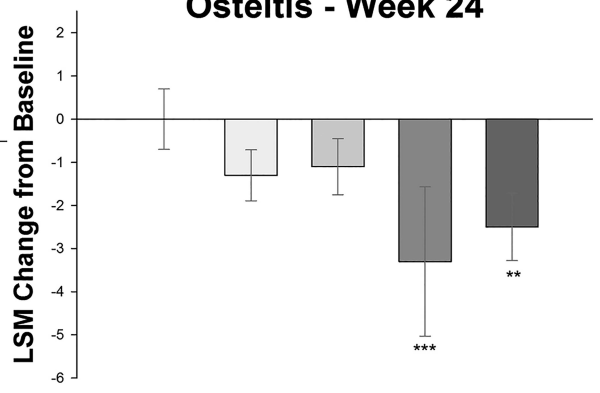

Figure 1. LSM change from baseline to Week 12 (left panels) and Week 24 (right panels) for (A) synovitis, (B) osteitis, and (C) combined inflammation scores. Significance values are based on ANCOVA. Error bars represent the standard error of the mean. $* \mathrm{P}<0.20$ vs placebo. $* * \mathrm{P}<0.05$ vs placebo. $* * * \mathrm{P}<0.01$ vs placebo. $* * * * \mathrm{P}<0.001$ vs placebo. LSM: least squares mean.

treatment with MTX. Similar to the reduction in signs and symptoms of RA, there was a dose-dependency to the responses of synovitis, osteitis, and combined inflammation, with effects being most pronounced for the baricitinib 4-mg and $8-\mathrm{mg}$ treatment arms. The proportion of patients with decreased synovitis provided supporting evidence for the dose association. Improvement in inflammation and inhibition of progression of joint damage continued over the second 12-week interval; however, direct comparisons with placebo were not possible for that interval because subjects in the placebo arm were rerandomized to baricitinib.

Based on the overall results of the phase IIb study, both the 2- and 4-mg doses demonstrated efficacy compared with placebo in terms of ACR20/50/70, low disease activity, and remission; the 4-mg dose provided greater efficacy and a more rapid onset of effect than the 2-mg dose. The 4-mg once-daily baricitinib dose was selected as the primary dose for phase III testing 9 . Results of the MRI substudy supported this dose selection and indicated that doses higher than $4 \mathrm{mg}$ were not required to achieve beneficial effects on osteitis or synovitis. These findings supported removal of the 8-mg dose from consideration and allowed introduction of a second, lower dose ( $2 \mathrm{mg}$ once daily) in clinical studies that did not include active comparators. Including the 2-mg dose allowed characterization of a minimally effective dose. In the 2 phase III studies with active comparators, the signs and symptoms of RA were improved with baricitinib $4 \mathrm{mg}$. Structural progression was inhibited, based on conventional radio-

Personal non-commercial use only. The Journal of Rheumatology Copyright (C) 2019. All rights reserved. 

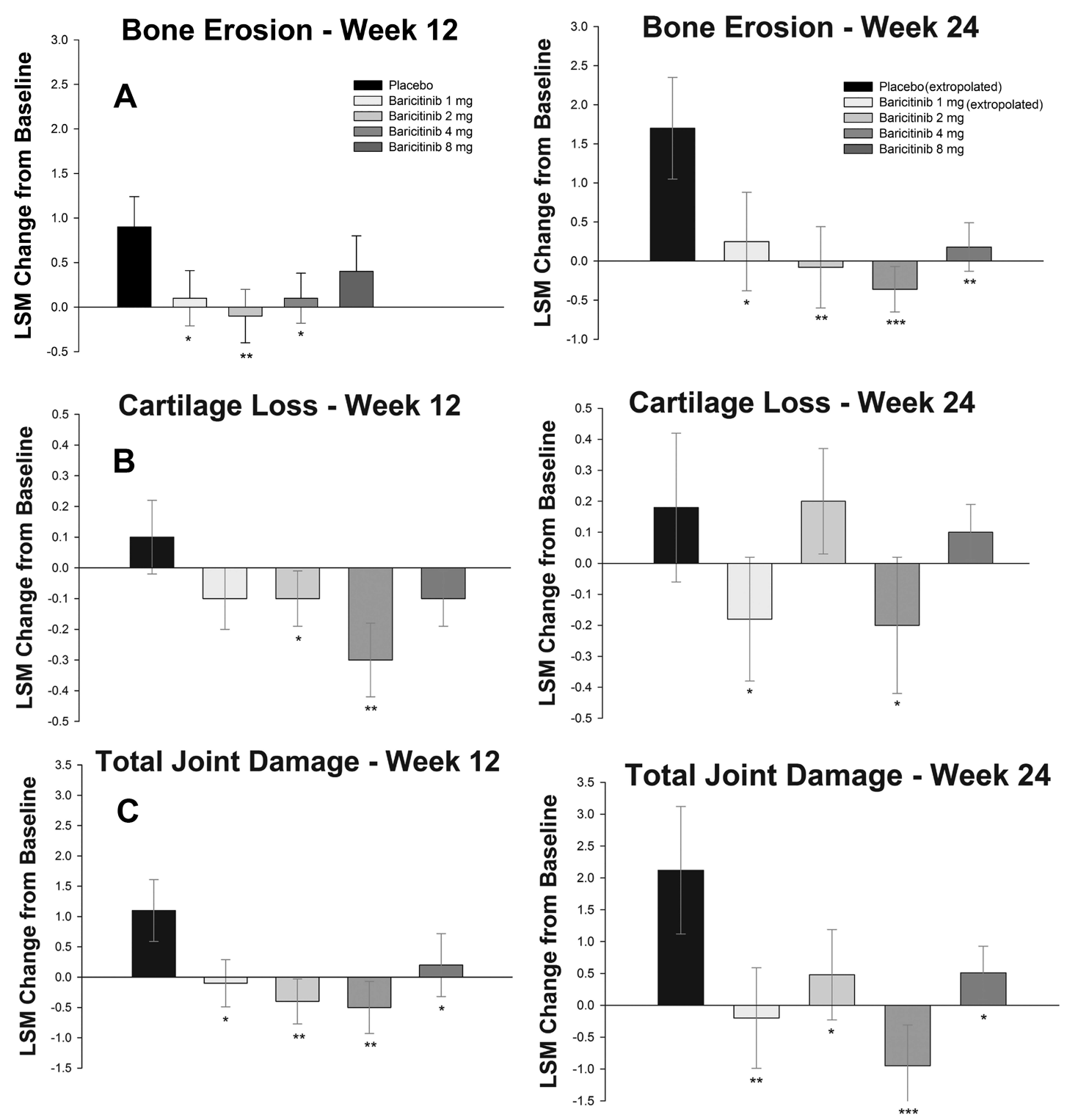

Figure 2. LSM change from baseline to Week 12 (left panels) and Week 24 (right panels) for (A) bone erosion, (B) cartilage loss, and (C) total joint damage scores. Significance values are based on ANCOVA. Error bars represent the standard error of the mean. $* \mathrm{P}<0.20$ vs placebo. $* * \mathrm{P}<0.05$ vs placebo. $* * * \mathrm{P}<0.01$ vs placebo. LSM: least squares mean.

graphy, in patients with active RA despite treatment with MTX (RA-BEAM) ${ }^{11}$ or who were naive to DMARD treatment $(\text { RA-BEGIN })^{12}$. In a third phase III study that included patients with active disease despite treatment with csDMARD (RA-BUILD) ${ }^{10}$, improved symptoms and function were observed, and exploratory data indicated reduction in structural damage progression with both doses of baricitinib. However, beneficial effects on joints were less pronounced with the $2-\mathrm{mg}$ dose, a finding that is consistent with the results of this MRI substudy.

Conventional radiography has been the accepted, standard imaging method for assessing the progression of joint damage in clinical trials. It has become increasingly challenging to ascertain differences in rates of progression of radiographic damage between treatment and control arms owing to lower disease activity among patients enrolled in clinical trials ${ }^{18,19}$, technological shift from film-based radiography to digital radiography ${ }^{5}$, and most importantly, limits on the duration of exposure to placebo or ineffective treatment for subjects with active $\mathrm{RA}^{20}$. Current regulatory guidance suggests that studies longer than 12 weeks include an active comparator as the control or provide rescue treatment for patients with active disease ${ }^{21}$. However, $\geq 24$ weeks is typically needed to demonstrate inhibition of structural progression by radiography in placebo-controlled trials, and even longer durations would be needed for active comparator trials or dose-ranging studies, which typically show smaller differences between treatment arms. Larger

Personal non-commercial use only. The Journal of Rheumatology Copyright ()$_{2019}$. All rights reserved. 


\begin{tabular}{|c|c|c|c|c|c|}
\hline & \multirow[b]{2}{*}{$\begin{array}{c}\text { Placebo, } \\
n=48\end{array}$} & \multicolumn{4}{|c|}{ Baricitinib } \\
\hline & & $\begin{array}{l}1 \mathrm{mg}, \\
\mathrm{n}=27\end{array}$ & $\begin{array}{l}2 \mathrm{mg} \\
\mathrm{n}=29\end{array}$ & $\begin{array}{l}4 \mathrm{mg}, \\
\mathrm{n}=26\end{array}$ & $\begin{array}{l}8 \mathrm{mg}, \\
\mathrm{n}=24\end{array}$ \\
\hline Patients with improvement in synovitis, $\mathrm{n}(\%)$ & $6(13)$ & $9(33)$ & $4(14)$ & $8(31)$ & $7(29)$ \\
\hline Uninvolved joints that developed new synovitis, mean $\%$ & 3.5 & 2.5 & 0.7 & 1.3 & 4.7 \\
\hline Patients with improvement in osteitis, $\mathrm{n}(\%)$ & $5(10)$ & $7(26)$ & $7(24)$ & $6(23)$ & $6(25)$ \\
\hline Uninvolved bones that developed new bone erosion, mean $\%$ & 2.7 & 0.3 & 0.6 & 0.9 & 1.1 \\
\hline Patients with no worsening in cartilage loss, $\mathrm{n}(\%)$ & $37(95)$ & $25(100)$ & $29(100)$ & $25(100)$ & $24(100)$ \\
\hline Uninvolved joints that developed new cartilage loss, mean $\%$ & 1.1 & 0.2 & 0.0 & 0.0 & 0.2 \\
\hline \multicolumn{6}{|l|}{ Week 24} \\
\hline Patients with improvement in synovitis, $\mathrm{n}(\%)$ & $4(8)$ & $7(26)$ & $5(17)$ & $8(31)$ & $8(33)$ \\
\hline Uninvolved joints that developed new synovitis, mean $\%$ & 3.5 & 2.5 & 2.2 & 6.4 & 2.6 \\
\hline Patients with no worsening in cartilage loss, $\mathrm{n}(\%)$ & $35(90)$ & $23(92)$ & $27(93)$ & $25(100)$ & $24(100)$ \\
\hline Uninvolved joints that developed new cartilage loss, mean $\%$ & 1.1 & 0.2 & 1.0 & 0.0 & 0.4 \\
\hline
\end{tabular}

Improvement in synovitis and in osteitis was defined as a change from baseline that exceeded the SDC. No worsening in bone erosion and in cartilage loss was defined as a change from baseline < SDC. Placebo and baricitinib 1-mg groups at Week 24 were imputed using last observation carried forward for synovitis and osteitis and using linear extrapolation for bone erosion and cartilage loss from baseline and Week 12 scores. MRI: magnetic resonance imaging; SDC: smallest detectable change.

sample sizes may offset some of this power loss, but to date no radiographic trial has reported inhibition of progression in only 12 weeks. These issues limit the utility of radiography, particularly in dose-ranging studies.

By using RAMRIS and CARLOS scoring, this study demonstrated the ability of MRI to discriminate treatment effects on inflammation and structural damage in RA within only 12 weeks in a trial with fewer than 50 patients per arm. When RAMRIS was initially described, it included assessment of synovitis, osteitis, and bone erosion, but it did not assess cartilage $\operatorname{loss}^{13}$. An MRI joint space narrowing (JSN) score has since been added to RAMRIS ${ }^{22}$. The CARLOS method was developed prior to RAMRIS-JSN and differs from it in that CARLOS assesses articular cartilage directly rather than indirectly on the basis of $\mathrm{JSN}^{14}$. Including a measure of cartilage loss allowed derivation of a total joint damage score by MRI, analogous to the radiographic total Sharp score that combines erosion and JSN scores to provide a more comprehensive assessment of joint damage in RA. To our knowledge, this is the first clinical trial to demonstrate that MRI can discriminate inhibition of progression of cartilage loss in RA within 12 weeks. Indeed, articular cartilage appeared to increase with treatment at 12 weeks and in the 4-mg and extrapolated 1 -mg arms at 24 weeks. Whether this represents actual regeneration of hyaline cartilage is not known, but if the finding is corroborated in future studies, it should be investigated further. A subsequent trial using CARLOS similarly showed inhibition of progression of cartilage loss in only 12 weeks ${ }^{23}$.

There are a number of limitations to these analyses. MRI assessments were obtained in only a subgroup of patients enrolled in the study, although randomization was stratified by subgroup participation. Because there were small numbers in each subgroup, we did not attempt to detect differences between active dose groups. Comparisons of changes in MRI-assessed features to those measured by conventional radiographs were not possible, because radiographs were not obtained over the same period because of the relatively short period of planned observation. Patients who participated in the substudy were required to have evidence of erosive disease; therefore, the effect of baricitinib on inflammation or the development of structural damage in patients with nonerosive RA was not assessed. Additionally, MRI changes at Week 24 for placebo and baricitinib 1-mg treatment arms were extrapolated from changes observed over the first 12 weeks, as patients in these treatment arms were rerandomized to higher doses of baricitinib to limit patient exposure to ineffective treatments. Statistical analyses were also not adjusted for multiplicity; therefore, some significant findings from this exploratory study could be false positives. Finally, among patients with baseline MR images who discontinued before the Week 12 assessment, a larger percentage were from the placebo group, thereby limiting the assessment of improvement in inflammatory measures in this group and becoming a potential source of bias, although sensitivity analyses not

Personal non-commercial use only. The Journal of Rheumatology Copyright $\subset$ (2019. All rights reserved. 
subject to this potential bias confirmed the findings in these measures.

In this phase IIb, dose-ranging study, use of MRI findings demonstrated dose-dependent suppression of synovitis and osteitis along with inhibition of progression of bone erosion within 12 weeks of initiating baricitinib, using a relatively small number of patients with active and erosive RA who showed inadequate response to treatment with MTX. When reviewed in the context of the clinical findings, these data informed dose selection for the phase III program. The available imaging data from this phase IIb study increased confidence that the 4-mg baricitinib dose would be able to demonstrate a positive effect on reducing the rate of radiographic progression in phase III studies.

\section{ACKNOWLEDGMENT}

The authors thank Stephanie C. Colvin, $\mathrm{PhD}$, and Victoria Crotzer, $\mathrm{PhD}$, of Eli Lilly and Co., and Kathy Oneacre, MA, of Syneos Health for medical writing support and assistance with preparation and submission of this manuscript.

\section{REFERENCES}

1. European Medicines Agency. Committee for Medicinal Products for Human Use. Guideline on clinical investigation of medicinal products other than NSAIDs for treatment of rheumatoid arthritis, draft. [Internet. Accessed March 18, 2019.] Available from: www.ema.europa.eu/docs/en_GB/document_library/Scientific_ guideline/2015/06/WC500187583.pdf

2. Aletaha D, Smolen JS. Joint damage in rheumatoid arthritis progresses in remission according to the Disease Activity Score in 28 joints and is driven by residual swollen joints. Arthritis Rheum 2011;63:3702-11.

3. Smolen JS, Van Der Heijde DM, St. Clair EW, Emery P, Bathon JM, Keystone E, et al; Active-Controlled Study of Patients Receiving Infliximab for the Treatment of Rheumatoid Arthritis of Early Onset (ASPIRE) Study Group. Predictors of joint damage in patients with early rheumatoid arthritis treated with high-dose methotrexate with or without concomitant infliximab: results from the ASPIRE trial. Arthritis Rheum 2006;54:702-10.

4. Baker JF, Ostergaard M, Emery P, Hsia EC, Lu J, Baker DG, et al. Early MRI measures independently predict 1-year and 2-year radiographic progression in rheumatoid arthritis: secondary analysis from a large clinical trial. Ann Rheum Dis 2014;73:1968-74.

5. Peterfy $\mathrm{C}, \varnothing$ stergaard $\mathrm{M}$, Conaghan PG. MRI comes of age in RA clinical trials. Ann Rheum Dis 2013;72:794-6.

6. American College of Rheumatology Rheumatoid Arthritis Clinical Trials Task Force Imaging Group and Outcome Measures in Rheumatology Magnetic Resonance Imaging Inflammatory Arthritis Working Group. Review: the utility of magnetic resonance imaging for assessing structural damage in randomized controlled trials in rheumatoid arthritis. Arthritis Rheum 2013;65:2513-23.

7. Conaghan PG, Durez P, Alten RE, Burmester GR, Tak PP, Klareskog L, et al. Impact of intravenous abatacept on synovitis, osteitis and structural damage in patients with rheumatoid arthritis and an inadequate response to methotrexate: the ASSET randomised controlled trial. Ann Rheum Dis 2013;72:1287-94.

8. Conaghan PG, Peterfy C, Olech E, Kaine J, Ridley D, Dicarlo J, et al. The effects of tocilizumab on osteitis, synovitis and erosion progression in rheumatoid arthritis: results from the ACT-RAY MRI substudy. Ann Rheum Dis 2014;73:810-6.

9. Keystone EC, Taylor PC, Drescher E, Schlichting DE, Beattie SD,
Berclaz PY, et al. Safety and efficacy of baricitinib at 24 weeks in patients with rheumatoid arthritis who have had an inadequate response to methotrexate. Ann Rheum Dis 2015;74:333-40.

10. Dougados M, van der Heijde D, Chen YC, Greenwald M, Drescher $\mathrm{E}$, Liu J, et al. Baricitinib in patients with inadequate response or intolerance to conventional synthetic DMARDs: results from the RA-BUILD study. Ann Rheum Dis 2017;76:88-95.

11. Taylor PC, Keystone EC, van der Heijde D, Weinblatt ME, Del Carmen Morales L, Reyes Gonzaga J, et al. Baricitinib versus placebo or adalimumab in rheumatoid arthritis. N Engl J Med 2017;376:652-62.

12. Fleischmann R, Schiff M, van der Heijde D, Ramos-Remus C, Spindler A, Stanislav M, et al. Baricitinib, methotrexate, or combination in patients with rheumatoid arthritis and no or limited prior disease-modifying antirheumatic drug treatment. Arthritis Rheumatol 2017;69:506-17.

13. Østergaard M, Peterfy C, Conaghan P, McQueen F, Bird P, Ejbjerg $\mathrm{B}$, et al. OMERACT Rheumatoid Arthritis Magnetic Resonance Imaging Studies. Core set of MRI acquisitions, joint pathology definitions, and the OMERACT RA-MRI scoring system. J Rheumatol 2003;30:1385-6.

14. Peterfy CG, DiCarlo JC, Olech E, Bagnard MA, Gabriele A, Gaylis $\mathrm{N}$. Evaluating joint-space narrowing and cartilage loss in rheumatoid arthritis by using MRI. Arthritis Res Ther 2012;14:R131

15. Markusse IM, Landewé R, Wolterbeek R, Ho M, Jenkins M, van der Heijde D. Linear extrapolation of missing radiographic change scores in clinical trials does not spuriously overestimate group radiographic changes in rheumatoid arthritis. Rheumatology 2016;55:1295-300.

16. Peterfy C, Emery P, Tak PP, Østergaard M, DiCarlo J, Otsa K, et al. MRI assessment of suppression of structural damage in patients with rheumatoid arthritis receiving rituximab: results from the randomised, placebo-controlled, double-blind RA-SCORE study. Ann Rheum Dis 2016;75:170-7.

17. Bruynesteyn K, Boers M, Kostense P, van der Linden S, van der Heijde D. Deciding on progression of joint damage in paired films of individual patients: smallest detectable difference or change. Ann Rheum Dis 2005;64:179-82.

18. Strand V, Sokolove J. Randomized controlled trial design in rheumatoid arthritis: the past decade. Arthritis Res Ther 2009;11:205.

19. Rahman MU, Buchanan J, Doyle MK, Hsia EC, Gathany T, Parasuraman $\mathrm{S}$, et al. Changes in patient characteristics in anti-tumour necrosis factor clinical trials for rheumatoid arthritis: results of an analysis of the literature over the past 16 years. Ann Rheum Dis 2011;70:1631-40.

20. American College of Rheumatology Rheumatoid Arthritis Clinical Trial Investigators Ad Hoc Task Force. American College of Rheumatology Clinical Trial Priorities and Design Conference, July 22-23, 2010. Arthritis Rheum 2011;63:2151-6.

21. U.S. Department of Health and Human Services, Food and Drug Administration. Guidance for industry: rheumatoid arthritis: developing drug products for treatment. [Internet. Accessed March 18, 2019.] Available from: www.fda.gov/downloads/drugs/guidancecomplianceregulatory information/guidances/ucm354468.pdf

22. Glinatsi D, Lillegraven S, Haavardsholm EA, Eshed I, Conaghan PG, Peterfy C, et al. Validation of the OMERACT magnetic resonance imaging joint space narrowing score for the wrist in a multireader longitudinal trial. J Rheumatol 2015;42:2480-5.

23. Beals C, Baumgartner R, Peterfy C, Balanescu A, Mirea G, Harabaglu A, et al. Treatment effects measured by dynamic contrast enhanced MRI and RAMRIS for rheumatoid arthritis [abstract]. Ann Rheum Dis 2013;72 Suppl 3:A748. 
APPENDIX 1. Efficacy outcomes at weeks 12 and 24.

\begin{tabular}{|c|c|c|c|c|c|}
\hline \multirow[t]{2}{*}{ Variables } & \multirow[b]{2}{*}{ Placebo, $\mathrm{n}=48$} & \multicolumn{4}{|c|}{ Baricitinib } \\
\hline & & $1 \mathrm{mg}, \mathrm{n}=27$ & $2 \mathrm{mg}, \mathrm{n}=29$ & $4 \mathrm{mg}, \mathrm{n}=26$ & $8 \mathrm{mg}, \mathrm{n}=24$ \\
\hline \multicolumn{6}{|l|}{ Week 12} \\
\hline DAS28-hsCRP & $-0.9(1.3)$ & $-1.4(1.4)$ & $-1.6(1.0)^{*}$ & $-2.2(1.1)^{* * *}$ & $-2.0(1.3)^{* * *}$ \\
\hline HAQ-DI & $-0.13(0.42)$ & $-0.30(0.57)$ & $-0.23(0.52)$ & $-0.33(0.48)$ & $-0.38(0.58) *$ \\
\hline Swollen joint count, 66 & $-6.0(7.9)$ & $-8.1(7.5)$ & $-7.5(8.1)$ & $-10.7(5.0)^{* * *}$ & $-10.3(6.6)^{* *}$ \\
\hline DAS28-hsCRP & - & - & $-1.7(0.9)$ & $-2.5(0.9)$ & $-2.3(1.2)$ \\
\hline HAQ-DI & - & - & $-0.24(0.47)$ & $-0.37(0.47)$ & $-0.50(0.52)$ \\
\hline Swollen joint count, 66 & - & - & $-9.4(7.6)$ & $-12.0(5.6)$ & $-12.1(5.3)$ \\
\hline
\end{tabular}

Data are LSM change from baseline (SD) unless otherwise indicated. Only patients in baricitinib 2-, 4-, and 8-mg treatment groups continued treatment for an additional 12 weeks. Significance values for ACR20 are based on 1-sided Fisher's exact test; significance values for DAS28-hsCRP, HAQ-DI, and swollen joint count (66) are based on ANCOVA. $* \mathrm{P}<0.05$ vs placebo. $* * \mathrm{P}<0.01$ vs placebo. $* * * \mathrm{P}<0.001$ vs placebo. ACR20: American College of Rheumatology criteria 20\% improvement; DAS28-hsCRP: 28-joint count Disease Activity Score based on high-sensitivity C-reactive protein; HAQ-DI: Health Assessment Questionnaire-Disability Index; LSM: least squares mean. 